\title{
Effects of Mixing-vane Attached to Grid Spacer on Pressure Drop and Deposition Rate in BWR Simulated Channel
}

\author{
M. Endo*, W. Takaki, D. Nishioka, Y. Hirakata, A. Kawahara, M. Sadatomi \\ Department of Mechanical System Engineering, Graduate School Science and Technology, Kumamoto University, Japan
}

Copyright $(2016$ by authors, all rights reserved. Authors agree that this article remains permanently open access under the terms of the Creative Commons Attribution License 4.0 International License

\begin{abstract}
This paper describes the experimental study on the effects of grid spacer with mixing-vane (MV) on gas-liquid two-phase annular flow in a vertical circular pipe of $16 \mathrm{~mm}$ i.d. In order to know the effects, grid spacers with and without MV were inserted in turn into the test channel. Furthermore, in order to know the effects of inclination angle of the mixing-vane to main flow, two inclination angles, i.e., $30^{\circ}$ (MV30) and $20^{\circ}$ (MV20) were tested. Pressure drop of flow through the spacer and liquid droplets deposition rate in the section downstream from the spacer were measured with a pressure transducer and double liquid film extraction method, respectively. Experimental data revealed that deposition mass transfer coefficient and the pressure drop were higher for the spacer with MV than that without MV. Regarding the effects of the inclination angle of MV, the vane of MV30 gave higher deposition mass transfer coefficient than MV20 although it increased the pressure drop.
\end{abstract}

Keywords Spacer, Mixing-vane, Liquid Film Thickness, Deposition

\section{Background and Introduction}

In a boiling water nuclear reactor (BWR), a coolant flow in a subchannel of the fuel rod assembly changes from a liquid single-phase flow to an annular two-phase flow, with flowing downstream. In the annular flow, a main part of the liquid phase flows as a liquid film on the fuel rod bundle and a remaining part flows as liquid droplets in the gas core. Since the liquid in the film evaporate and a part of that entrains as the droplets to the gas core by a high shear gas flow in the gas core, the thickness of the liquid film gradually decreases with flowing downstream. If the liquid film disappears, the rod temperature goes up and finally delivers damage to the fuel rod. In order to prevent this, the liquid film must be maintained enough for cooling the fuel rod.
Researches have been conducted recently on the mechanism of liquid film thickness recovery by the deposit of the liquid droplets entrained in the gas core to the liquid film by a mixing-vane (MV) attached to a grid spacer. A CFD-code, which can analyze flows in detail, has been developed to predict the droplets transfer. It is essential to validate the code with reliable experimental data. However, the published data on the effects of the spacer with MV is limited (e.g. Damsohn and Prasser [1]; Feldhaus et al. [2]).

The purpose of this series of studies is to provide such data by conducting experiments on the effects of the mixing-vane on the liquid droplets deposition in gas-liquid annular flows. As the test channel, a circular pipe simplifying a center subchannel in the fuel rod assembly was used. Grid spacers with and without MV were installed in turn into the test channel. For the spacer with MV, the inclination angle of four vanes to main flow was changed as 20 and 30 degrees. The pressure drop of flow through the spacer and the liquid droplets deposition rate in the section downstream from the spacer were measured. A similar experiment was conducted in our previous study (Kawahara et al. [3]) by adopting a wall liquid injection method, in which water was injected from a perforated channel wall to air flow as a liquid film. In the present study, contrary to this, we adopted a center jet injection method, in which water is introduced from a nozzle hole placed at the center of the channel to air flow as liquid droplets to increase the liquid droplets fraction, because the fraction in the gas core in actual BWR is considered to be higher due to lower surface tension.

\section{Experimental Methods}

\subsection{Test Apparatus}

Fig. 1 (a) shows a schematic diagram of the test apparatus. A vertical circular pipe of $D=16 \mathrm{~mm}$ i.d. was used as the test channel. Water and air at room temperature were used as the working fluids. The water was fed from a tank (\#6) to an 
air-water mixer (\#1) by a pump (\#7). As the mixer, as shown in Fig. 1 (b), a nozzle with a $3 \mathrm{~mm}$ i.d. hole at the center of the channel was inserted to inject water as a liquid jet. Then, the water jet was sheared by a flow of air supplied from a compressor (\#10), and broken down into liquid droplets in the air flow. The air-water mixture flowed upwards through an entry section (\#2), a test section (\#3) and a discharge section (\#4), and flowed into a separator (\#5). The water separated here was returned to the tank and reused, while the air was released to the atmosphere. The volume flow rate of the water was measured with an electromagnetic flow meter (\#8) within the accuracy of $\pm 2 \%$ and that of the air with an ultrasonic flow meter (\#9) within the accuracy of $\pm 5 \%$. In the test section, one of the test spacers was installed, and the pressure drop of flow through the spacer and the liquid droplets deposition rate in the section downstream from the spacer were measured.
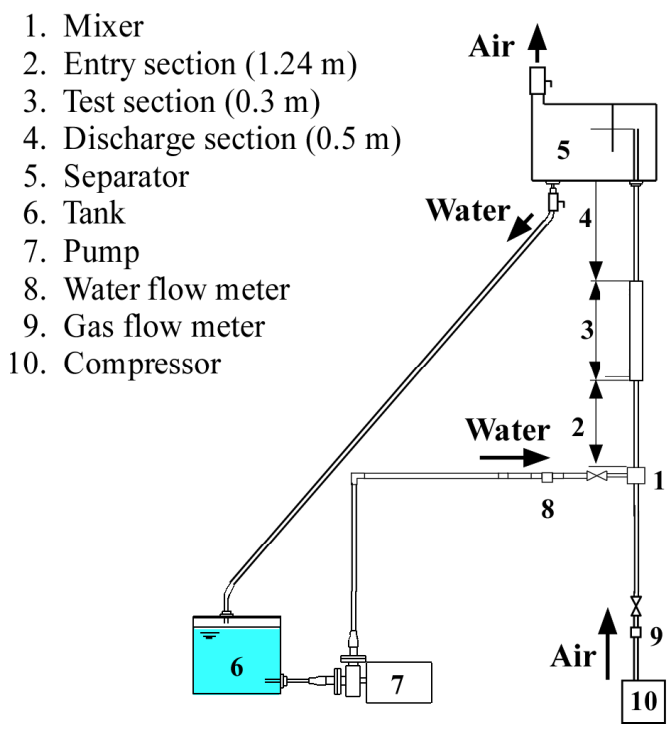

(a) Test apparatus
Fig. 2 shows three kinds of grid spacers. Fig. 2 (a) is the spacer without MV, and two $0.5 \mathrm{~mm}$ thick and $37.5 \mathrm{~mm}$ long plates made of PET (polyethylene terephthalate) were orthogonally connected each other. Fig. 2 (b) is the spacer with MV. Four vanes were attached to the upper end of the grid spacer. The vanes were inclined at 30 degree or 20 degree from the flow axis to create swirl flow. The test spacer with the mixing-vane having the inclination angle of $\theta$ $=30^{\circ}$ and $20^{\circ}$ are called as the spacer with MV30 and MV20, respectively.

\subsection{Measurement of Deposition Rate}

In this experiment, deposition rate was measured with liquid film extraction method. Fig. 3 shows a detail of the liquid film extraction units in the test section. Two extraction units were installed in two sections upstream and downstream from the spacer.

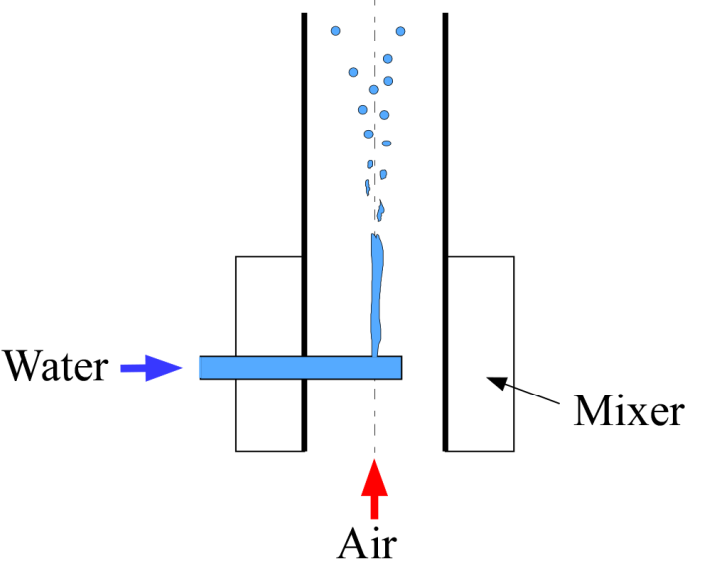

(b) Mixer

Figure1. Experimental apparatus
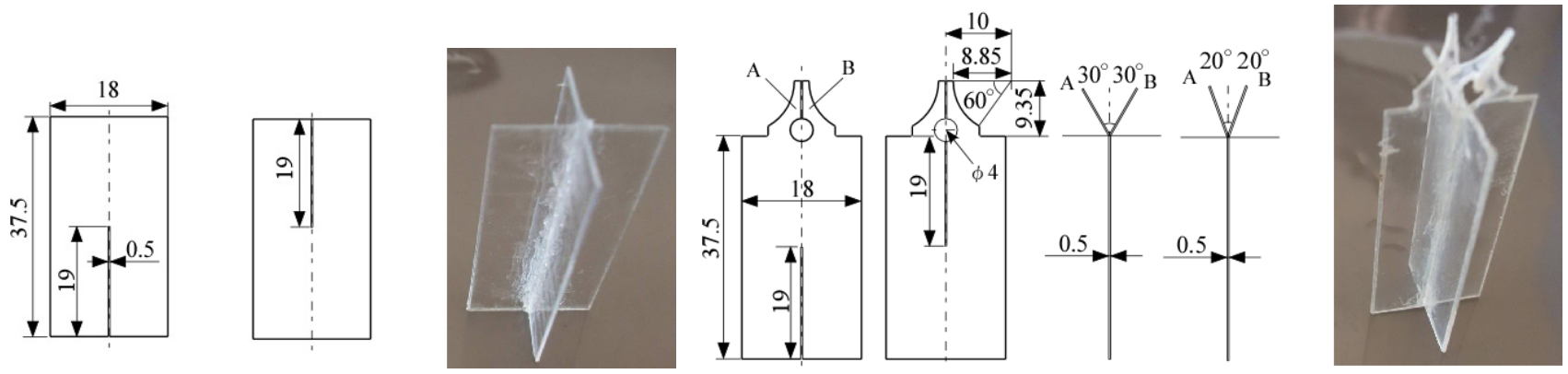

(a) Spacer without MV

(b) Spacer with MV $\left(\theta=30^{\circ}\right.$ or $\left.20^{\circ}\right)$

Figure 2. Test spacers 


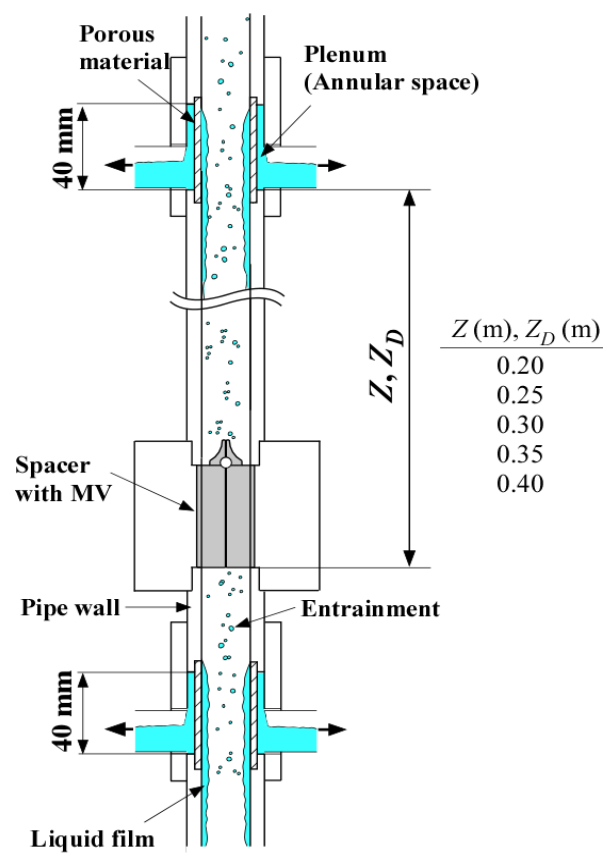

Figure 3. Measurement of deposition rate with liquid film extraction

The deposition rate of liquid droplets per unit surface area of the pipe wall, $m_{D}$, is usually evaluated as

$$
m_{D}=K C \text {. }
$$

Here, $K$ is the mass transfer coefficient and $C$ is the mean concentration of liquid droplets entrained in the gas core defined as:

$$
C=\frac{G_{L E}}{j_{G}+G_{L E} / \rho_{L}}
$$

where $j_{G}$ is the gas volumetric flux, $\rho_{L}$ is the liquid density, and $G_{L E}$ is the mass flux of entrained liquid droplets per cross-sectional area of the channel.

By substituting the present experimental data on $G_{L E}$ at two liquid film extraction sections etc. into (3) (Govan et al.,
[4]), we could determine the experimental data of $K$.

$$
K=\frac{D}{4 Z_{D}} \frac{G_{G}}{\rho_{G}} \ln \frac{G_{L E 1}}{G_{L E 2}},
$$

where $Z_{D}$ is the deposition length, $\rho_{G}$ is the gas density, $G_{G}$ (= $\left.\rho_{G} j_{G}\right)$ is the mass flux of gas introduced to the test channel. Here, $G_{L E 1}$ and $G_{L E 2}$ were given by the following conservation equations:

$$
\begin{gathered}
G_{L E 1}=G_{L}-G_{L F 1}, \\
G_{L E 2}=G_{L E 1}-G_{D},
\end{gathered}
$$

where $G_{L}$ is the mass flux of liquid introduced to the test channel, and $G_{D}$ is the mass flux of liquid droplets deposited to the wall after passing through the first liquid film extraction unit.

In order to know axial variations in $K$ with $Z_{D}, Z_{D}$ was changed from $0.2 \mathrm{~m}$ to $0.4 \mathrm{~m}$, as seen in Fig. 3 .

In addition, the experimental data of the fraction of liquid droplets in the gas core, i.e., entrainment fraction, $E$, was determined by substituting $G_{L}$ and $G_{L F 1}$ data into:

$$
E=\frac{G_{L}-G_{L F 1}}{G_{L}}
$$

\subsection{Measurement of Pressure Drop}

Fig. 4 (a) shows a pressure drop measurement system. Pressure taps were located at eight positions indicated by \#1 to \#8, and connected to a gauge type pressure transducer with the accuracy of $4 \%$. Fig. 4 (b) shows typical example of pressure distribution data for the spacer with MV30 in annular flow at liquid and gas volumetric fluxes of $j_{L}=0.05$ $\mathrm{m} / \mathrm{s}$ and $j_{G}=25 \mathrm{~m} / \mathrm{s} . P_{\text {in }}$ and $P_{\text {out }}$ were gauge pressures at the upstream and the downstream ends of the spacer extrapolated from pressure distributions upstream and downstream from the spacer. The pressure drop by the spacer, $\Delta P_{S}$, was determined by the pressure difference $\left(=P_{\text {in }}-P_{\text {out }}\right)$.

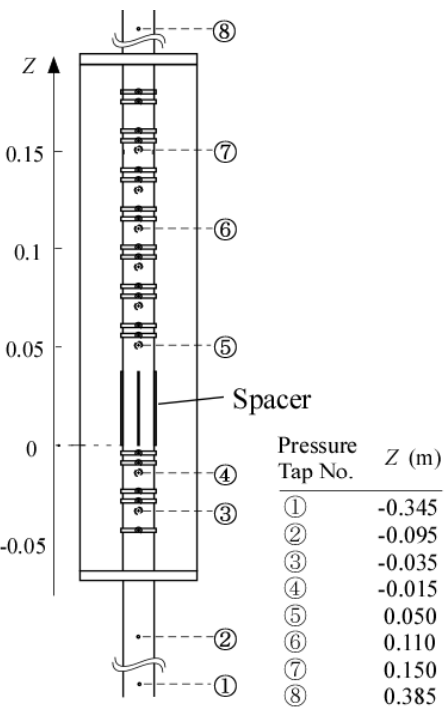

(a) Apparatus

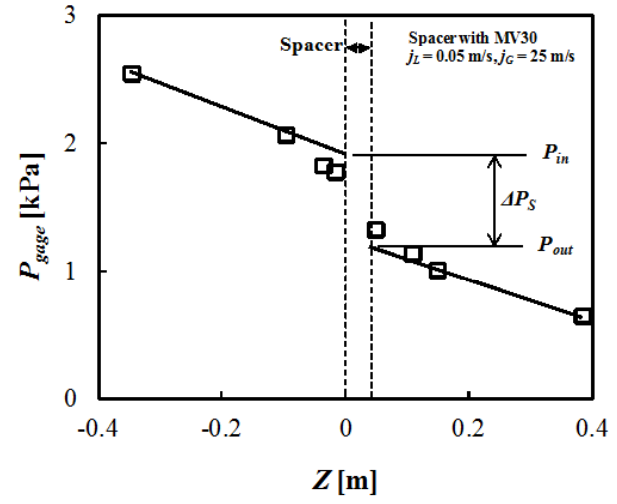

(b) Pressure distribution along the channel axis

Figure4. Determination of pressure drop 


\section{Results and Discussion}

\subsection{Entrainment Fraction}

Fig. 5 shows the data on the entrainment fraction, $E$, plotted against the gas volumetric flux, $j_{G}$ for no spacer. The dotted line represents the calculation by Oliemans et al. [5], which is based on AERE Harwell data bank. The present $E$ data for the center jet injection are $24 \%$ higher than those for the wall injection by Kawahara et al. [3]. The calculation agrees with $E$ data for the wall injection, but under-predict them for the center jet injection.

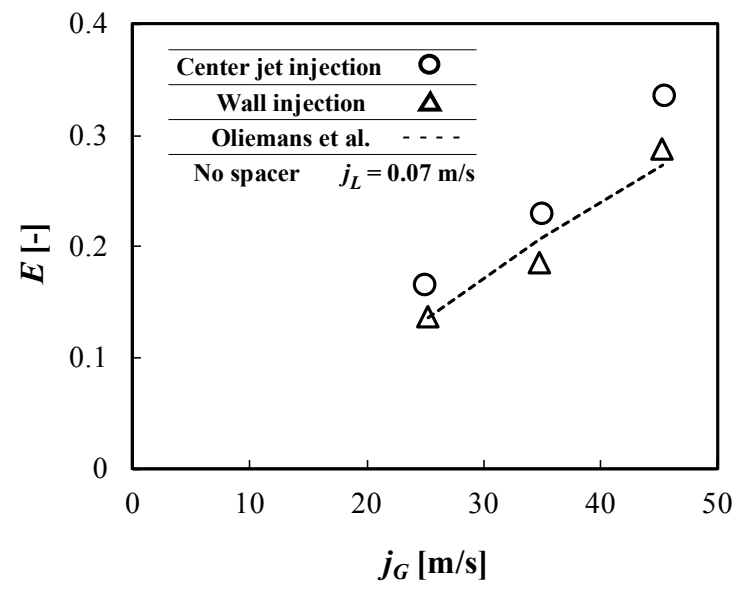

Figure 5. The data on liquid entrainment fraction

\subsection{Deposition Rate}

Fig. 6 shows the dimensionless mass transfer coefficient, $K^{*}$, data obtained at $Z=0.2 \mathrm{~m}$, plotted against the dimensionless mean droplet concentration, $C^{*}$. $K^{*}$ and $C^{*}$ are defined as:

$$
\begin{gathered}
K^{*}=K \sqrt{\frac{\rho_{G} D}{\sigma}}, \\
C^{*}=\frac{C}{\rho_{G}} .
\end{gathered}
$$

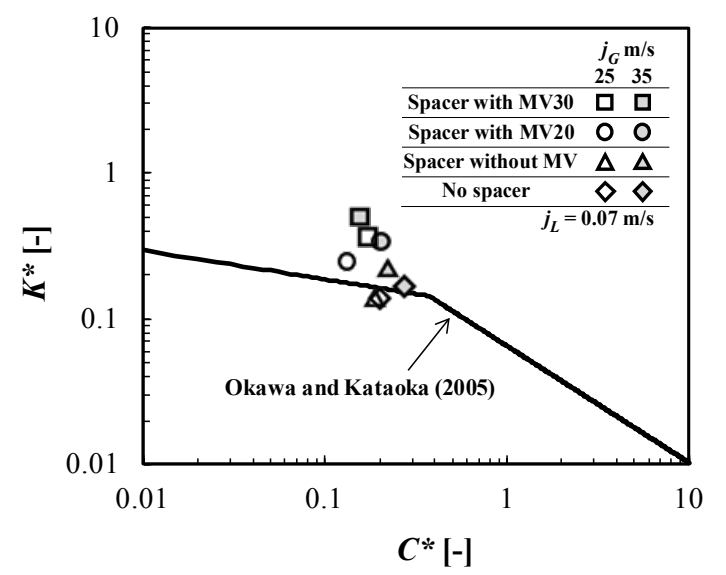

Figure 6. Dimensionless deposition mass transfer coefficient data obtained at $Z=0.2 \mathrm{~m}$, versus dimensionless mean droplet concentration
The solid line represents the calculated value by Okawa and Kataoka's correlation [6]. $K^{*}$ data for no spacer are close to the calculation, i.e., the calculation is valid for the center jet injection case. For the spacer without $\mathrm{MV}, K^{*}$ values also lie close to the calculation. For the spacer with $\mathrm{MV}$, on the other hand, $K^{*}$ data are higher than the calculation, because mixing-vane creates a swirl flow, causing centrifugal force on droplets to deposit to the liquid film. In addition, $K^{*}$ data are higher for the MV30 than MV20.

Fig. 7 shows the deposition mass transfer coefficient data plotted against the axial distance from the upstream end of the spacer, $Z$, at $j_{L}=0.07 \mathrm{~m} / \mathrm{s}$ and $j_{G}=35 \mathrm{~m} / \mathrm{s}$. We can notice the following trends: (a) $K$ data for no spacer are almost constant, independent of $Z$; (b) $K$ data for the spacer without $\mathrm{MV}$ are slightly higher than those for no spacer due to gas flow turbulence induced by the grid spacer inserted in the test channel; (c) $K$ data are much higher for spacer with MV than those without MV, because the swirl flow induced by the vane increases droplets deposition to liquid film; (d) $K$ data are higher for MV30 than MV20, i.e., larger inclination angle makes stronger swirl flow; (e) The effects of swirl flow created by the vane become weak with flowing downstream; (f) $K$ data for the spacer with MV20 are almost the same as those for the spacer without $\mathrm{MV}$ at $Z=0.4 \mathrm{~m}$, while $K$ data for MV30 is $49 \%$ higher.

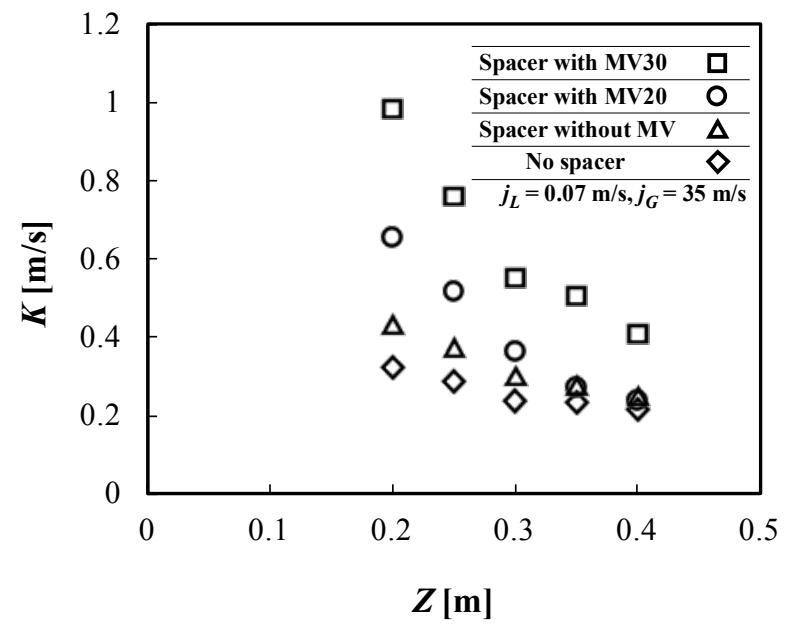

Figure 7. Axial variations in deposition mass transfer coefficient $\left(j_{L}=\right.$ $0.07 \mathrm{~m} / \mathrm{s}, j_{G}=35 \mathrm{~m} / \mathrm{s}$ )

\subsection{Pressure Drop}

Fig. 8 shows experimental data on the pressure drop by the spacer, $\Delta P_{S}$, plotted against the gas volumetric flux, $j_{G}$.

$\Delta P_{S}$ increases with $j_{G}$ and $j_{L}$ for all types of spacers. $\Delta P_{S}$ is about 37\% higher for the spacer with MV30 than that without MV. $\triangle P_{S}$ is about $24 \%$ higher for MV30 than MV20, because blockage ratio, $\varepsilon_{S}$, i.e., the ratio of projected area of the $\mathrm{MV}, A_{S}$, to total flow area, $A$, is lager for MV30 $\left(\varepsilon_{S}=\right.$ $0.279)$ than MV20 $\left(\varepsilon_{S}=0.191\right)$. From Figs. 7 and 8 , it is found that the spacer with MV30 can give the highest liquid droplet deposition rate, but causes the largest pressure drop. 


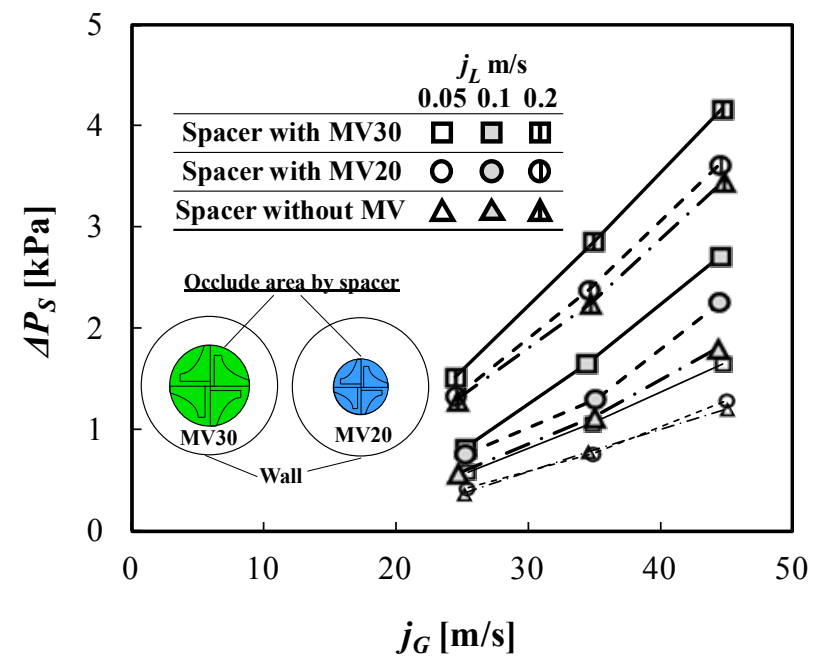

Figure 8. Pressure drop by the spacer

Data on pressure loss coefficient by a spacer, $\zeta_{S}$, were obtained by substituting $\Delta P_{S}$ data into the next equation:

$$
\zeta_{S}=\frac{\Delta P_{S}}{\rho_{H} j_{G}^{2} / 2}
$$

Here, $\rho_{H}$ is the homogeneous density in the gas core and given by:

$$
\rho_{H}=\frac{\rho_{G} Q_{G}+\rho_{L} Q_{L E}}{Q_{G}+Q_{L E}},
$$

where $Q_{G}$ and $Q_{L E}$ are the volume flow rates of gas and liquid droplets, respectively.

Fig. 9 shows the resulting $\zeta_{S}$ data against the gas volumetric flux, $j_{G}$.

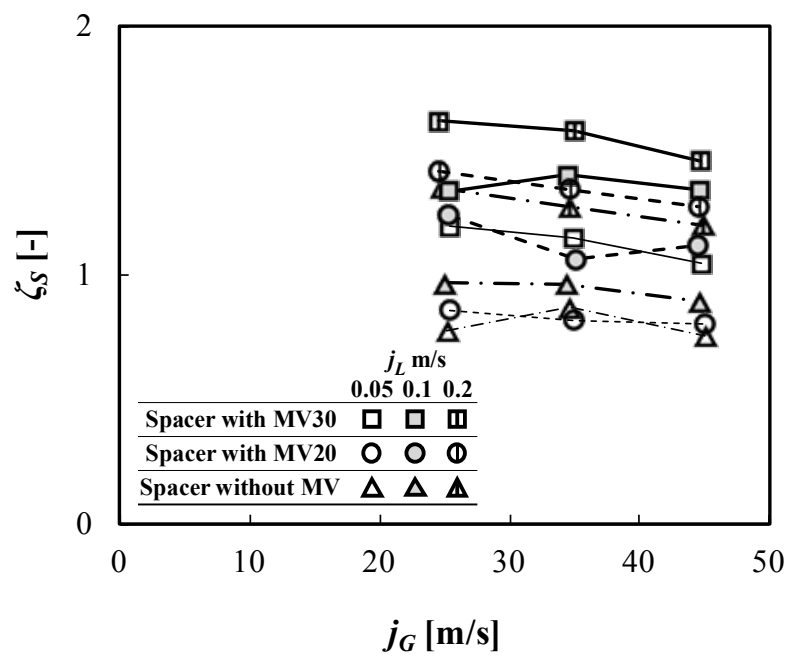

Figure 9. Pressure loss coefficient for two-phase annular flow

$\zeta_{S}$ are almost constant independent of $j_{G}$ but depend of spacer type. In addition, $\zeta_{S}$ increases with $j_{L}$ at a fixed $j_{G}$. Except for $j_{L}=0.05 \mathrm{~m} / \mathrm{s}$ and $j_{G}=35 \mathrm{~m} / \mathrm{s}, \zeta_{S}$ is higher for the spacer with MV than that without MV, because kinetic energy of flow is consumed to create swirl flow. Thus, $\zeta_{S}$ is higher for the spacer with MV30 than that with MV20.
In our previous study (Kawahara et al. [3]), the ratio of the spacer pressure loss coefficient for two-phase annular flow to that for single-phase gas flow, $\zeta_{S, T P} / \zeta_{S, S P}$, for the liquid wall injection case was correlated with the following equation:

$$
\zeta_{S, T P} /_{\zeta_{S, S P}}=1+121(1-\beta) \text {. }
$$

where $\beta$ is the gas volume flow rate fraction $\left(=j_{G} /\left(j_{G}+j_{L}\right)\right)$. Fig. 10 shows the experimental data on $\zeta_{S, T P} / \zeta_{S, S P}$ against the liquid volume flow fraction, $1-\beta$. Solid line represents the calculation by Eq. (11). The calculation agrees well with the present data with $6.84 \%$ in mean error and $13.40 \%$ in the root-mean-square error, respectively. Thus, Eq. (11) is valid for calculating $\zeta_{S, T P} / \zeta_{S, S P}$ irrespective of the difference in the liquid injection method and the entrainment fraction at the spacer position.

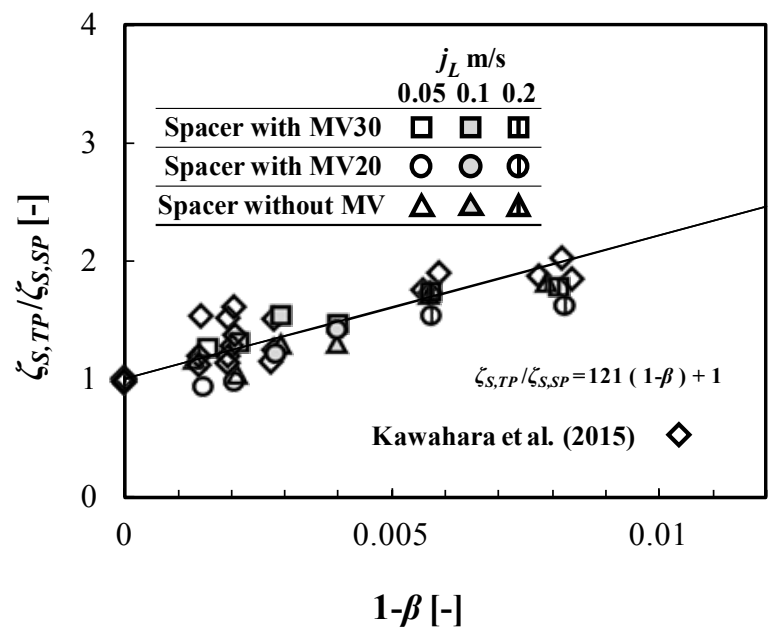

Figure10. Ratio of pressure loss coefficient for two-phase flow to single-phase one

\section{Conclusions}

The effects of mixing-vane (MV) attached to a grid spacer on the characteristics of air-water annular flows were experimentally investigated using a $16 \mathrm{~mm}$ i.d. circular pipe as the test channel, which was simplified from the center subchannel in a BWR fuel rod bundle. In the test, water was injected from the pipe center to increase the liquid entrainment fraction while it was injected from the pipe wall in our previous study. To know the effects of inclination angle of MV to main flow, two angles, $\theta=30^{\circ}$ (MV30) and $20^{\circ}$ (MV20), were tested. The main findings are as follows:

(1) The entrainment fraction was higher for the present center jet water injection than the wall water injection.

(2) The mixing-vanes effectively promoted droplet deposition to liquid film on the channel wall, because the mass transfer coefficient was higher for the spacer with MV than that without MV.

(3) Effects of the mixing-vane on flow continued to a finite axial distance from the spacer, depending on 
the inclination angle of the mixing-vane. That is, the axial distance affecting the vane increased with the inclination angle of the vane.

(4) Pressure drop by the spacer was the highest for the spacer with MV30, because the blockage ratio for MV30 was the largest.

(5) The ratio of the spacer pressure loss coefficient for two-phase annular flow to that for single-phase gas flow are correlated with a linear function of liquid volumetric flow rate fraction proposed in our previous study for the wall water injection case.

\section{REFERENCES}

[1] M. Damsohn, H.-M. Prasser. Experimental studies of the effect of functional spacers to annular flow in subchannels of a BWR fuel element, Nuclear Engineering and Design, Vol.
240, No. 10, 3126-3144, 2010.

[2] G. Feldhaus, B. J. Azzopardi, W. Zeggel. Annular flow experiments in rod bundles with spacers, Nuclear Engineering and Design, Vol. 213, Nos. 2-3, 199-207, 2002.

[3] A. Kawahara, M. Sadatomi, S. Imamura, Y. Shimoharai, Y. Hirakata, M. Endo. Effects of grid spacer with mixing vane on entrainment and depositions in two-phase annular flows, Nuclear Engineering and Technology, Vol. 47, No. 4, 389-397, 2015.

[4] A. H. Govan, G. F. Hewitt, D. G. Owen, T. R. Bott. An improved CHF modeling code, Proceedings of the second UK National Heat Transfer Conference, 33-48, 1988.

[5] R. V. A. Oliemans, B. F. M. Pots, N. Trompe. Modeling of annular dispersed two-phase flow in vertical pipes, Int. J. Multiphase Flow, Vol. 12, 711-732, 1986.

[6] T. Okawa, I. Kataoka. Correlations for the mass transfer rate of droplets in vertical upward annular flow, Int. J. Heat and Mass Transfer, Vol. 48, Nos. 23-24, 4766-4778, 2005. 